\title{
Potential use of the Upper Eyelid Myocutaneous Flap in the Reconstruction of Full-thickness Defects of the Medial Canthus
}

\section{Alshehri Sarah ${ }^{1}$, Lauwers Fréderic ${ }^{2,3}$ and Lopez Raphael ${ }^{2,3 *}$ \\ ${ }^{1}$ Department of Otorhinolaryngology, Purpan Hospital, Toulouse, France ${ }^{2}$ Department of Maxillo-Facial Surgery, Purpan Hospital, Toulouse, France \\ ${ }^{3}$ Laboratory of Anatomy Purpan, Faculty of Medicine, Toulouse, France}

\section{Case Report}

We report our method in the reconstruction of two cases of fullthickness defects of the medial canthal region treated after radical excision of a malignant cutaneous lesion in the medial canthus (basal cell carcinoma or squamous cell carcinoma) and adjuvant radiotherapy. In our department of Plastic and Maxillo-facial Surgery Department of Purpan Hospital. In both cases, the reconstruction was performed on two levels, using two local flaps.

\section{Anatomy}

The Upper Eyelid Myocutaneous Flap (UEMF) was removed in the preseptal zone of the orbicular muscle. Figure 1 illustrates the axial vascularity of the upper eyelid flap after the carotid injection with coloured latex and shows the upper eyelid arch (preseptal arch) [1] located above the orbital septum plane.

If we follow the arterial blood supply [2], we can separate two UEMFS: One with the medial pedicle emerging from the medial lid artery. And the second with the superior medial pedicle emerging from the superior orbital or trochlear artery. Thus, in the cases of canthus tissue loss with a damage of the medial eyelid artery, an upper eyelid flap with a superior medial pedicle could be used instead (Figure 2).

\section{Surgical Technique}

The surgical technique involves the performance of a one-stage procedure resulting in two flaps that provide two-level reconstruction of the medial canthus full-thickness defect.

An UEMF with a medial pedicle [3] ensures a deep plane reconstruction. The design of the incision is made in the upper eyelid fold to minimise the aesthetic effects. The eyelid resection involves the

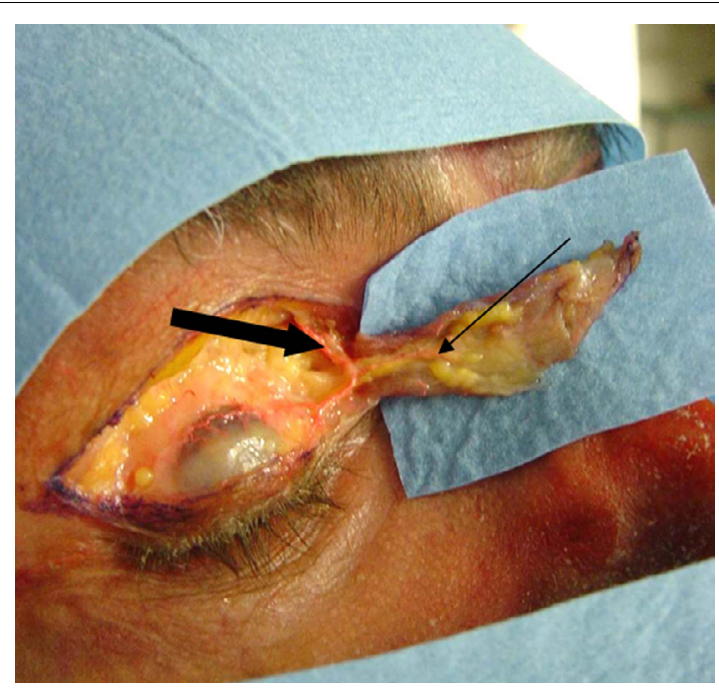

Figure 1: The vascularization of the upper eyelid myocutaneous flap by the upper eyelid arch, after injection of Neoprene Latex: (large arrow: supero medial pedicle, thin arrow: upper lid arch).

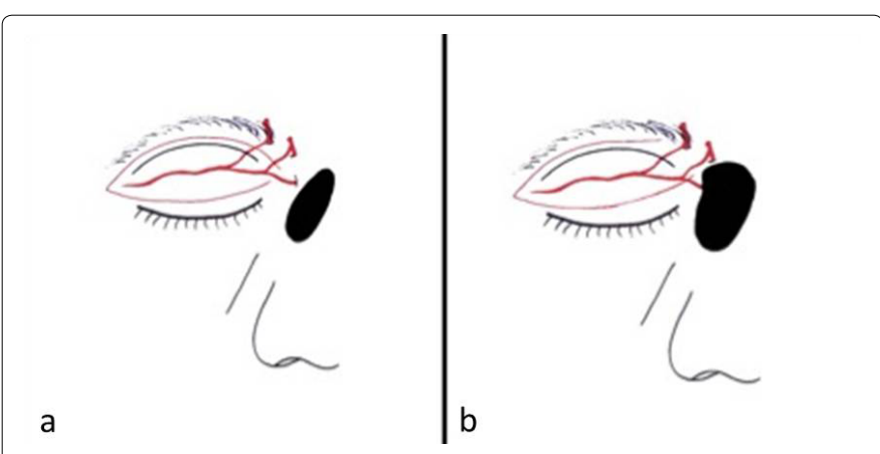

Figure 2a: Diagram of the medial pedicled upper eyelid flap. In the case of canthus tissue loss damaging the medial lid artery, this flap can't be used. b: Diagram of the supero medial pedicled upper eyelid flap. This flap could be used in spite of all.

preseptal portion of the orbicular muscle. The dissection is performed behind the orbital septum, allowing the elevation of the myocutaneous flap from the lateral to the medial face, which ensures a maximal blood supply (Figure 3). A tunnel is made to slide the flap towards the deep plane of the full-thickness defect. A de-epidermisation of the tunnel is subsequently performed. The superficial plane reconstruction is performed using a cutaneous local flap, i.e., median or para-median forehead flap, glabellar flap, bilobed flap, or rhomboidal flap. A contralateral blepharoplasty is performed during the same operation to provide a symmetric eyelid appearance.

\section{Patient 1}

A 70-year-old woman presented a sclera dermiform basal cell carcinoma of the left medial canthus. After a radical resection and adjuvant radiotherapy with iridium 192 was performed in the Oncology Clinical Center. Following a year of rigorous clinical supervision, a medial forehead flap reconstruction was performed for her. Three months later she had a healing defect and she developed an orbito-nasal fistula (Figure 4a). She was operated few weeks later for a revision repair surgery; a repair of both planes was performed, using a UEMF for the deep plane and a nasolabial flap with lateral pedicles for the superficial plane.

The immediate postoperative outcomes were simple, and 15 days

*Corresponding author: Lopez Raphaël, Plastic and Maxillo-facial Surgery Department, CHU Purpan, Place du Dr Baylac, F-31059 Toulouse Cedex, France, Tel: +33 5617725 17; Fax: +33 5617791 23; E-mail: lopez.r@chu-toulouse.fr

Received February 05, 2014; Accepted February 18, 2014; Published February 27,2014

Citation: Sarah A, Fréderic L, Raphael L (2014) Potential use of the Upper Eyelid Myocutaneous Flap in the Reconstruction of Full-thickness Defects of the Medial Canthus. Surgery Curr Res 4: 182. doi:10.4172/2161-1076.1000182

Copyright: ( 2014 Sarah A, et al. This is an open-access article distributed under the terms of the Creative Commons Attribution License, which permits unrestricted use, distribution, and reproduction in any medium, provided the original author and source are credited. 


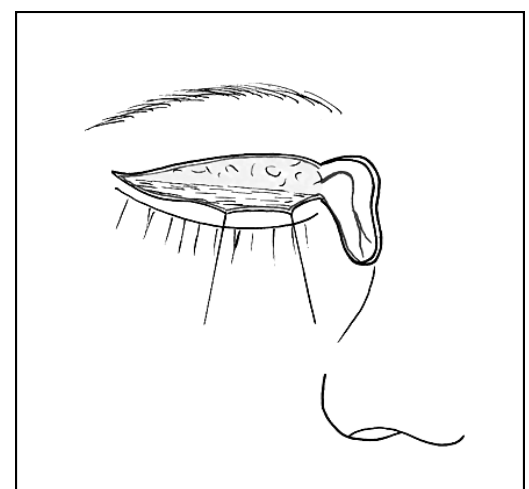

Figure 3: The UEMF with a medial pedicle ensures a deep plane reconstruction.

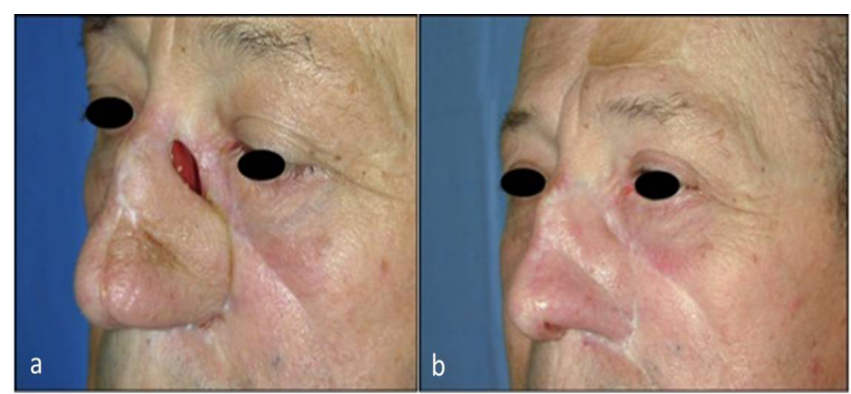

Figure 4a: The full thickness defect of the medial canthus. Figure b: Early post-operative result.

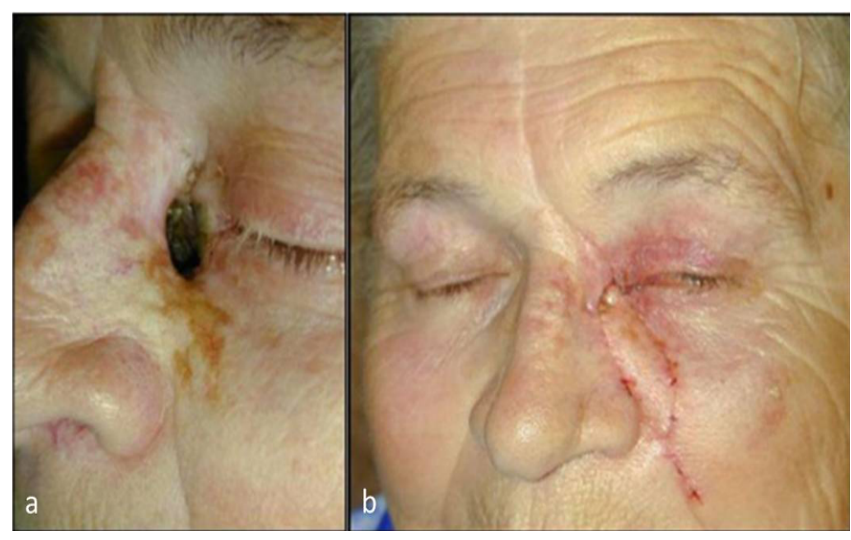

Figure 5a: Medial canthus defect despite previous forehead flap. b: Two years post-operative aesthetic result.

later, the aesthetic and functional postoperative results were satisfactory (Figure 4b).

\section{Patient 2}

A 63-year-old man was referred to our unit to evaluate a recurrent squamous cell carcinoma on the left side of his nose, which required a partial rhinectomy and external adjuvant radiotherapy. More than a year later, a reconstruction by a converse scalping flap was performed with satisfactory aesthetic and functional results. More than a year after the surgery, the patient developed an orbito-nasal fistula in the left medial canthal region (Figure 5a)

The reconstruction technique consisted of a UEMF with medial pedicle and a rotation flap of the previous forehead flap (Figure 5b).

A contralateral blepharoplasty for eye appearance symmetry was performed. The long-term (two years later) aesthetic and functional results were satisfactory, without recurrence of the orbito-nasal fistula.

\section{Discussion}

The other etiological factors of orbito-nasal osteonecrosis reported in the literature are the result of infections, particularly rhinocerebral mucormycosis [4].

Many reconstruction techniques for the periorbital region have been described previously and their limited applications evoked the necessity of a consensus approach for these techniques [5]. Spinelli and jelks [5] proposed an algorithm for the reconstruction of the periorbital region after dividing it into different zones. Accordingly, they define the internal canthal region as zone III.

The majority of these surgical techniques are applicable to the repair of superficial tissue loss. To the best of our knowledge, only one article reports the reconstruction of full-thickness defects of the medial canthus [6].

An upper eyelid flap with medial or superior medial pedicle, whose first anatomical description was reported in 1961 by Corso [7], can also be used to perform this reconstruction.

By following the dominant artery for the vascularisation of the upper eyelid flap, Borman and ozcan [2] differentiated two types of flaps with the medial pedicle: the UEF-MP (Upper Eye Flap-Medial Pedicle) for small tissue losses of the medial canthal region and the UEF-SMP (Upper Eye Flap-Super Medial Pedicle) for more significant tissue losses.

The UEMF presents many advantages such as the simplicity of the technique, the good vitality of vascularity, the respect of aesthetics units, and the absence of morbidity from the donor site. These advantages can lead some surgeons to choose this flap for superficial tissue loss of the medial canthal region [3].

The contraindications in performing this surgical technique are: craniofacial disjunction (Le Fort III fractures), medial orbital fracture, scar where the medial pedicle would be destroyed and exophthalmos.

Only one study in the literature describes an interest in using the upper eyelid in the endonasal reconstruction of full-thickness defects of the nose involving three planes of rehabilitation [8]. These authors underline advantages that make it particularly well-adapted to endonasal reconstruction. None of the studies, however, showed any interest in this flap for the endonasal plane reconstruction of fullthickness defects of the medial canthal region.

\section{Conclusion}

The myocutaneous upper eyelid flap is used in two cases to repair the deep plane of medial canthus full-thickness defects, and it has several advantages, particularly in orbito-nasal osteoradionecrosis, which increase its indications in the medial canthus reconstruction.

\section{References}

1. Lopez R, Lauwers F, Paoli JR, Boutault F, Guitard J (2008) The vascular system of the upper eyelid. Anatomical study and clinical interest. Surg Radiol Anat 30: 265-269.

2. Borman H, Ozcan G (2001) Superomedially based upper eyelid musculocutaneous flap for closure of medial canthal defects. Eur J Plast Surg 24: $239-242$. 
Citation: Sarah A, Fréderic L, Raphael L (2014) Potential use of the Upper Eyelid Myocutaneous Flap in the Reconstruction of Full-thickness Defects of the Medial Canthus. Surgery Curr Res 4: 182. doi:10.4172/2161-1076.1000182

3. Jelks GW, Glat PM, Jelks EB, Longaker MT (2002) Medial canthal reconstruction using a medially based upper eyelid myocutaneous flap. Plast Reconstr Surg 110: $1636-1643$.

4. Lari AR, Kanjoor JR, Vulvoda M, Katchy KC, Khan ZU (2002) Orbital reconstruction following sino-nasal mucormycosis. Br J Plast Surg 55: 72-75.

5. Spinelli HM, Jelks GW (1993) Periocular reconstruction: a systematic approach. Plast Reconstr Surg 91: 1017-1024.

6. Putterman AM (1989) Reconstruction of nasal fistulas of the medial canthus. Am J Ophthalmol 108: 68-74.
7. Corso PF (1961) Variations of the arterial, venous, and capillary circulation of the soft tissues of the head by decades as demonstrated by the methyl methacrylate injection technique and their application to the construction of flaps and pedicles. Plast Reconstr Surg 27:160-184.

8. Capizzi PJ, Eaves FF, Kane WJ (1999) The transverse orbicularis oculi myocutaneous flap: its use as nasal lining. Plast Reconstr Surg 104: 14191423 